\title{
Branching Ratio for the Rare Pion Decay into Positron and Neutrino
}

\author{
G. Czapek, A. Federspiel, A. Flückiger, D. Frei, B. Hahn, C. Hug, E. Hugentobler, W. Krebs, U. Moser, \\ D. Muster, E. Ramseyer, H. Scheidiger, P. Schlatter, and G. Stucki \\ Laboratorium für Hochenergiephysik, University of Berne, CH-3012 Bern, Switzerland
}

\author{
R. Abela, D. Renker, and E. Steiner \\ PSI (Paul Scherrer Institut), CH-5232 Villigen, Switzerland
}

(Received 24 July 1992)

\begin{abstract}
A new precision measurement of the branching ratio of the rare pion decay into a positron and a neutrino $(\pi \rightarrow e v)$ has been completed. A beam of positive pions was stopped in an active target of plastic scintillator surrounded by a $4 \pi$ BGO calorimeter. $3 \times 10^{5}$ rare decays and $1.2 \times 10^{6}$ normal pion decays $(\pi \rightarrow \mu \nu)$ were recorded. The branching ratio was finally calculated from $1.2 \times 10^{5}$ rare decays after various cuts in the time window from 7.5 to $200 \mathrm{~ns}$ after pion stop. The errors of the result $(1.235 \pm 0.005) \times 10^{-4}$ are $0.28 \%$ statistical and $0.29 \%$ systematical.
\end{abstract}

PACS numbers: $13.20 . \mathrm{Cz}$

An experiment was performed to measure the branching ratio $R$ for the rare decay $\pi \rightarrow e v(\gamma)$ (i.e., $\pi \rightarrow e v$ including $\pi \rightarrow e v \gamma$ ), normalized to the normal pion decay $\pi \rightarrow \mu v(\gamma)$ with high precision, testing the $\mu e$ universality of the weak interaction. This rare decay has been treated theoretically many times and values for $R$ have been published by several authors during the development of the theory of the weak interaction [1-3] and also later in the framework of gauge theories. The most recent predictions for $R$ are as follows:

$$
\begin{aligned}
& \text { Marciano and Sirlin [4], }(1.233 \pm 0.004) \times 10^{-4} ; \\
& \text { Goldman and Wilson [5], }(1.239 \pm 0.001) \times 10^{-4} \text {; } \\
& \text { Marciano [6], }(1.2345 \pm 0.0010) \times 10^{-4} \text {. }
\end{aligned}
$$

First experimental attempts to measure $R$ were made more than forty years ago [7] but it was only in 1958 when the rare decay could really be seen $[8,9]$ and a first result for $R$ was published in 1960 [10]: $R=(1.21$ $\pm 0.07) \times 10^{-4}$. Later the uncertainty was pushed down to about $2 \%$ by $\mathrm{Di}$ Capua et al. [11]: $R=(1.247$ $\pm 0.028) \times 10^{-4}$. This value has been updated to $1.273 \times 10^{-4}$ using the latest value for the pion lifetime [12]. An experiment at TRIUMF by Bryman et al. led to a more accurate result [13]: $R=(1.218 \pm 0.014)$ $\times 10^{-4}$. An improved version of the same experiment produced a significantly better result [14]:

$$
R=[1.2265 \pm 0.0034 \text { (stat) } \pm 0.0044 \text { (syst) }] \times 10^{-4} \text {. }
$$

Our experiment was done at SIN (PSI). The analysis of the data sample has now been completed and a result for the branching ratio has been obtained:

$$
R=[1.2346 \pm 0.0035 \text { (stat) } \pm 0.0036 \text { (syst) }] \times 10^{-4} \text {. }
$$

Comparing the latest theoretical result by Marciano [6] with our measurement one finds for the ratio of the electroweak coupling constants $g_{e} / g_{\mu}=1.000 \pm 0.002$, confirming $\mu e$ universality. The TRIUMF value agrees with our result within the quoted errors.

The experiment (Fig. 1) was set up in the $\pi \mathrm{M} 3$ area at PSI. Positive pions were stopped in the plastic scintillator target $T$ at a distance of $25 \mathrm{~m}$ from the pion production target. The beam contained a substantial contamination of muons and electrons. The electrons could easily be suppressed by an electrostatic separator to about $1 \%$ of the pion flux. The amount of muons at the end of the beam line was about equal to the number of pions. The muons could be discriminated in the trigger using the time-of-flight information relative to the accelerator $\mathrm{rf}$.

The pions which survived the degrading phase came to rest in $T$ and subsequently decayed. Pion interactions in flight were not considered, only pion decays inside a time window from 7.5 to $200 \mathrm{~ns}$ after the pion stop were selected. The branching ratio $R$ is equal to the number of $\pi \rightarrow e v(\gamma)$ decays divided by the number of $\pi \rightarrow \mu v(\gamma)$ decays in this time interval.

A $\pi \rightarrow e v(\gamma)$ event produced a first pulse in $T$ from the stopping pion followed by a second one from the outgoing positron in coincidence with an electromagnetic shower in the surrounding BGO calorimeter of an average thickness of 18 radiation lengths.

The dominant background process was the pion decay into muon and neutrino followed by the radiative decay of the muon ( $\pi \mu e$ chain). It produced a first pulse in $T$ from the stopping pion and a second one from the decay $\pi \rightarrow \mu v(\gamma)$ where the $4-\mathrm{MeV}$ muon came to rest after about $1 \mathrm{~mm}$ flight path in $T$. The muon decay caused a third pulse in $T$ in coincidence with a shower in the calorimeter.

In principle the rare decay mode could be selected just by discriminating the positron energy in the calorimeter, which is $70 \mathrm{MeV}$ for the $\pi \rightarrow e v$ decay and up to $53 \mathrm{MeV}$ (Michel spectrum) for the muon decay. Unfortunately the situation becomes more complicated because of the radiative muon decay where the sum of the positron and photon energies extends up to the muon rest mass and the fact that the measured energy spectrum of the $70-\mathrm{MeV}$ 
shower has a low-energy tail which reaches under the muon decay spectrum. So we could not use a simple trigger just by discriminating the energy of the decay products. We decided to set up the following two different triggers running simultaneously.

(i) The $\pi \rightarrow e v$ trigger which suppressed as many of the background events as possible without affecting the rare $\pi \rightarrow e v$ events. The aim of this trigger was to consider every stopping pion as a candidate for one of the rare decays unless it was a clearly identified background event. Setting an energy threshold helped to reduce background triggers, but one had to correct for the low-energy tail of the $\pi \rightarrow e v$ signal. Accepting only decays up to $200 \mathrm{~ns}$ after pion stop was the first important step to suppress muon decay background, because only $10 \%$ of the muons decayed before that time. A sequence of three pulses in the stopping target was an obvious sign for a background event. Thus the trigger reduced the background by a factor of 300 ; i.e., we had to accept 30 triggers per $\pi \rightarrow e v$ event.

(ii) The normalization trigger allowed us to determine the total number of pion decays inside the same gate from 7.5 to $200 \mathrm{~ns}$. The statistical uncertainty of the final result for the branching ratio was dominated by the number of accumulated events for the rare decay. Therefore it was sufficient to record only every 2000 th pion stop for the normalization.

The main components of the detector are shown in Fig. 1. The beam of about 5000 positive pions per second was defined by the last trigger counter $S$ with an area of $12 \times 12 \mathrm{~mm}^{2}$ and stopped inside of $1 \mathrm{~cm}^{3}$ in the center of the cylindrical active target $T$ (NE Pilot U) with a diameter of $38 \mathrm{~mm}$ and a height of $36 \mathrm{~mm}$. Before the experiment the spatial distribution of the pion stops had been measured with a small bubble chamber. The first $50 \mathrm{~ns}$ of the target signal were recorded using a wave-form digi-

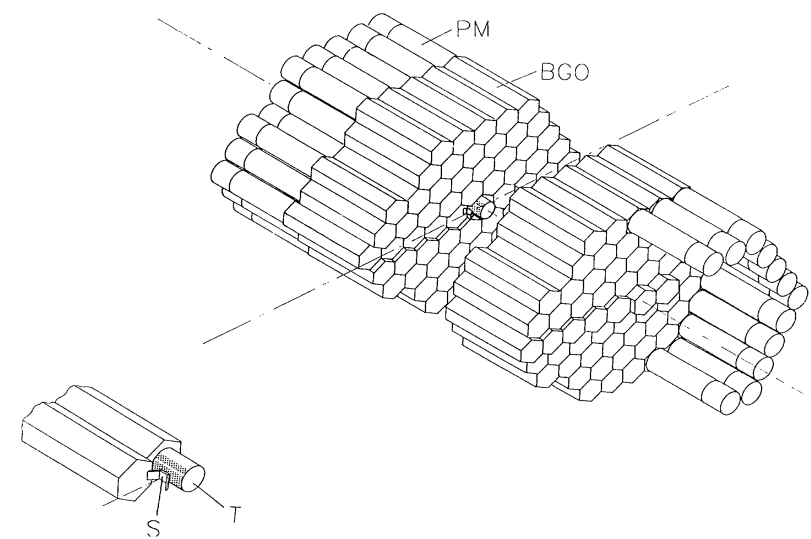

FIG. 1. Exploded view of the experimental setup. Shown are the last beam defining scintillator $S$, the active $\operatorname{target} T$, and the BGO calorimeter. A single BGO crystal has a length of $20 \mathrm{~cm}$ and an inner diameter of $5.5 \mathrm{~cm}$. tizer (Tektronix 7912 AD) with a sampling rate of 10 $\mathrm{GHz}$. The target light was read out through the two central BGO crystals which served as light guides for the target light and as elements of the calorimeter at the same time, leading to an excellent energy resolution of the target of better than $\pm 2.5 \%$ at $20 \mathrm{MeV}$ and minimizing the inactive material inside the electromagnetic calorimeter. The loss of solid angle acceptance of the calorimeter was less than $0.2 \%$ and only due to the entrance hole for the particle beam. For a $4 \pi$ calorimeter the effect of backscattering of electrons from the calorimeter surface can be neglected (for an arrangement where the calorimeter only covers a limited fraction of the total solid angle the corrections for electron backscattering might reach the $1 \%$ level). The calorimeter was made of 132 identical BGO crystals $(20 \mathrm{~cm}$ long, hexagonal shape, $5.5 \mathrm{~cm}$ diameter) each with a 2-in. photomultiplier. For every trigger, the pulse height and pulse time of each individual crystal were recorded. The light yield of a single crystal was homogeneous over its whole length to within $1.5 \%$. The intercalibration of the 132 elements was done on line with cosmic ray muons. As the calorimeter was segmented into independent elements it was possible to do a very detailed cross-check of its uniformity in time and pulse height. The electron energy resolution of the calorimeter was $\pm 1.7 \%$ at $90 \mathrm{MeV}$. The time information for the positron showers was good to $\pm 1 \mathrm{~ns}$. The whole setup was surrounded by a cosmic anticounter made of a layer of $2.5-\mathrm{cm}$ plastic scintillator shielded on the inside by $5 \mathrm{~cm}$ of lead to prevent self-vetoing of showers coming from the BGO calorimeter. In order to avoid pileup a dead time of $20 \mu \mathrm{s}$ (nine muon lifetimes) was introduced for every particle entering the detector.

The main data were taken during a 14-week run in the summer of 1988 . A total of $2.4 \times 10^{9}$ pions were accumulated; $3 \times 10^{5}$ of the rare $\pi \rightarrow e v(\gamma)$ decays and $1.2 \times 10^{6}$ $\pi \rightarrow \mu v(\gamma)$ decays were recorded. Figure 2 shows a twodimensional histogram of the energy deposited in the target and the total energy of the $\pi \rightarrow e v$ trigger events. The total energy includes the kinetic energy of the pion $(20 \mathrm{MeV})$ and the energy of all the decay products in the active target and the calorimeter. Before the target and calorimeter energies could be summed the quenching for the different particles in the target scintillator had to be corrected for. For the typical $\pi \rightarrow e v(\gamma)$ decay the total energy equals $90 \mathrm{MeV}$ and the target energy $24 \mathrm{MeV}$ (the positron energy loss peaks at $4 \mathrm{MeV}$ ). The totalenergy distribution of the $\pi \mu e$ chain background events, $\pi \rightarrow \mu v(\gamma)$ followed by $\mu \rightarrow e v v(\gamma)$, falls off sharply at $77 \mathrm{MeV}$ whereas the target energy peaks at about 28 $\mathrm{MeV}$ (due to additional $4 \mathrm{MeV}$ muon kinetic energy). The two processes can be distinguished nicely.

At the cost of losing a factor of 2 in statistics, additional very stringent off-line cuts were applied to the pionstop defining parameters. This reduced the beam correlated background significantly without favoring either of 


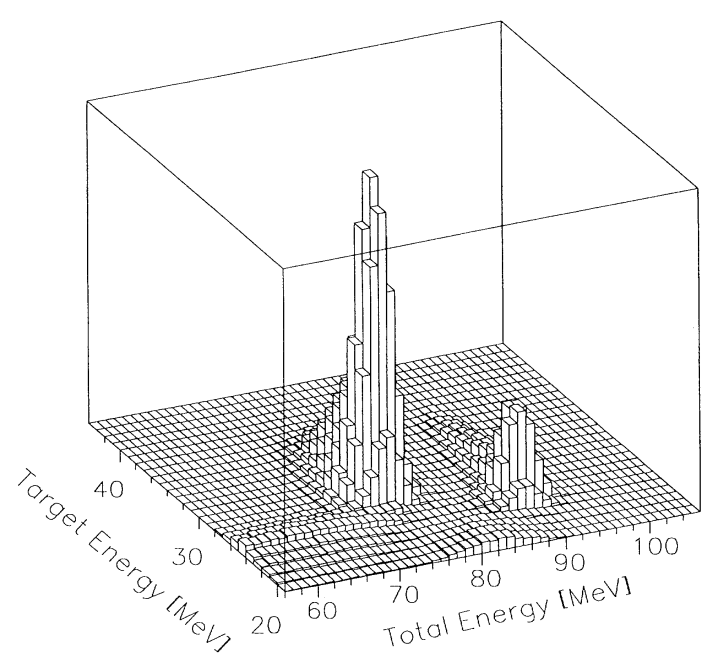

FIG. 2. Raw data of $\pi \rightarrow e v$ trigger events. The $\pi \rightarrow e v$ peak at $90 \mathrm{MeV}$ total energy and $24 \mathrm{MeV}$ target energy is clearly separated from the $\pi \mu e$ chain background events.

the two decay modes. The resulting total energy spectrum is shown in Fig. 3. There are three main contributions to the spectrum, namely, the $\pi \rightarrow e v(\gamma)$ signal, remaining $\mu$ decays, and a contribution from pion strong interactions which had not been rejected by the hardware trigger. In order to determine the number of $\pi \rightarrow e v(\gamma)$ events the spectrum of Fig. 3 was divided into three regions, the window from 83.5 to $101 \mathrm{MeV}$ containing most of the $\pi \rightarrow e v(\gamma)$ events and the two regions above and below which are dominated by background (the lower bound of the energy window was chosen in order to minimize the final error). The low-energy region contains $\pi \mu e$ chain background events whereas the high-energy region contains mainly pion reactions with the target material. The shapes of the two background spectra were obtained with dedicated triggers enhancing these processes without distorting their energy spectra. Figure 3 shows the contributions of these backgrounds to the counts in the $\pi \rightarrow e v$ interval after proper normalization.

To the number of $\pi \rightarrow e v(\gamma)$ events in the central window we had to add the number of $\pi \rightarrow e v(\gamma)$ events in the low-energy tail below the window. To this end the electromagnetic shower propagation was simulated with the CERN EGS-4 code taking into account all the geometrical details of the detector. The precision of $0.2 \mathrm{MeV}$ of the total-energy calibration at the $\pi \rightarrow e v$ peak position at 90 $\mathrm{MeV}$ leads to an uncertainty for the resulting branching ratio of $0.08 \%$. A modified version of the CERN GEANT code was developed to evaluate the shower energy leakage due to nuclear photoabsorption with neutron emission. The main contribution comes from absorption on bismuth, which has a cross section of about $500 \mathrm{mb}$ [15] at the giant resonance energy around $13 \mathrm{MeV}$. Virtual processes like $\left(e, e^{\prime} n\right)$ are suppressed $[O(\alpha)]$ and there-

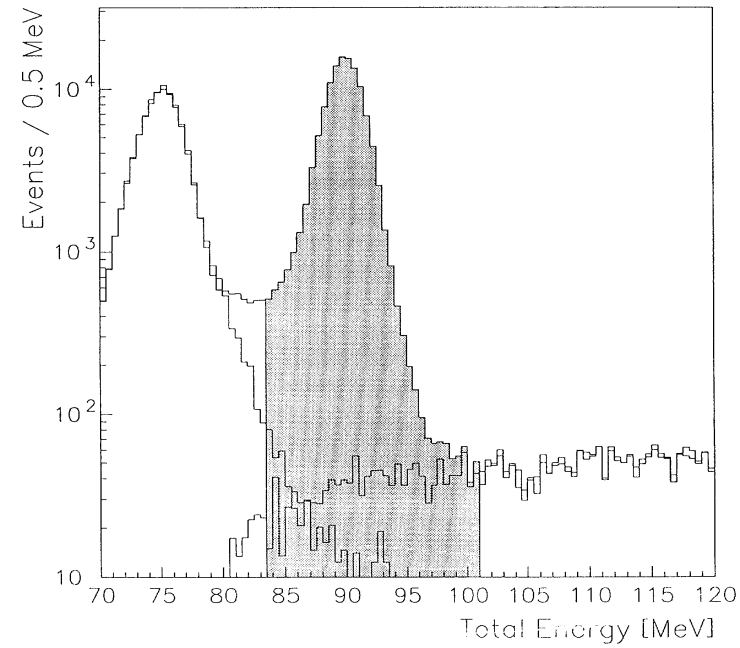

FIG. 3. The total-energy spectrum after applying cuts. Marked is the window used to calculate the branching ratio. The contributions from the $\pi \mu e$ chain background and the pion strong interaction background are shown.

fore neglected. If all the energy of the produced neutrons were lost this would result in an additional loss of $1.4 \%$ of the $\pi \rightarrow e v$ events falling below the cut energy of 83.5 $\mathrm{MeV}$. The estimation of the fraction of the neutron energy which is reabsorbed in the calorimeter is properly done in the GEANT code as we have convinced ourselves. Looking at the cross sections and the $Q$ values of all the possible neutron reactions it became clear that the only two substantial contributions come from $(n, \gamma)$ and $(n, \alpha)$ on $\mathrm{Bi}$. Including the partial reabsorption of the neutron energy reduced the correction due to photonuclear absorption to $0.95 \%$.

The correction for $\pi \rightarrow e v(\gamma)$ events above the cut of $101 \mathrm{MeV}$ total energy was smaller than $5 \times 10^{-4}$ and is therefore not discussed any further.

A lot of care was put into the analysis of the target signal. The time difference between the pion stop and the subsequent decays was obtained by fitting a series of measured standard pulse shapes to the measured target signal to get the time and the amplitude of each individual pulse. The crucial point was not to introduce any systematic time difference in the treatment of muon and positron signals. An error of 26 ps would have affected the result for the branching ratio by $0.1 \%$. The difference in the time distributions of the light production due to the different flight paths of decay muons and positrons is 21 ps and has been corrected for. The dimensions of the target have been chosen such as to obtain similar pulse heights for muons and positrons (about $4 \mathrm{MeV}$ ) to keep the systematic differences small.

The correction for positron pulses not detected in the active target $(0.15 \%)$ due to bremsstrahlung or annihila- 
tion was easy to make as the efficiency was directly measured by just selecting positrons with the calorimeter and looking at the energy loss spectrum in the target. The similar correction for decay muons is at least 10 times smaller and therefore neglected.

Our final result for the branching ratio is

$$
\begin{aligned}
R & =\Gamma(\pi \rightarrow e v(\gamma)) / \Gamma(\pi \rightarrow \mu v(\gamma)) \\
& =(1.235 \pm 0.005) \times 10^{-4} .
\end{aligned}
$$

The quoted error consists of $0.28 \%$ statistics and $0.29 \%$ systematics. The statistical error is a substantial contribution to the overall uncertainty of the result. During the analysis of the data it had become clear that the safe procedure was to apply stringent cuts to a series of parameters to minimize the systematic errors. The starting time of the measuring gate ( 7.5 to $200 \mathrm{ns)}$ was quite critical. We chose $7.5 \mathrm{~ns}$ to be certain that we could resolve the two target pulses from pion stop and pion decay well enough to efficiently detect the $\pi \rightarrow \mu v(\gamma)$ decays on the cost of losing the $25 \%$ of the pion decays before $7.5 \mathrm{~ns}$.

A listing of the main corrections and systematic errors is given in Table $\mathrm{I}$. The three most important corrections are the ones for losses of $\pi \rightarrow e v$ events due to electromagnetic shower leakage and nuclear photoabsorption and the one introduced by the subtraction of the remaining background events from radiative $\mu$ decay as described above.

Looking at the errors, it is obvious that there is room for improvement, first by running for several months at higher beam intensities to reduce the statistical error to about $0.1 \%$. At this level of precision the systematic errors become dominant. The most difficult problem seems to be the further reduction of the $\pi \mu e$ chain background. To this end a finely structured stopping target, which allows one to distinguish the different particles involved, might be developed. A still more precise investigation of the low-energy tail of the $\pi \rightarrow e v$ spectrum will be necessary maybe in connection with additional test experiments with the BGO calorimeter in an electron beam. The EGS simulation showed that the chosen thickness of the BGO calorimeter of 18 radiation lengths on average was well adapted to have about equal losses due to the beam entrance hole and to shower leakage through the BGO.

The authors want to thank K. Borer, M. Hess, L. Martinez, and the collaborators of the electronics and technical workshops of the LHEP of the University of Berne as well as the crew of the PSI Hallendienst for their excellent support. We thank F. Jegerlehner from PSI and J.
TABLE I. Main corrections and systematic errors of our final result for the branching ratio for the rare decay $\pi$ $\rightarrow e v(\gamma)$.

\begin{tabular}{lcc}
\hline \hline \multicolumn{1}{c}{ Process } & $\begin{array}{c}\text { Correction } \\
(\%)\end{array}$ & $\begin{array}{c}\text { Error } \\
(\%)\end{array}$ \\
\hline Electromagnetic losses & +1.64 & 0.09 \\
Photonuclear reactions & +0.95 & 0.19 \\
$\pi \rightarrow e v \gamma$ events with $E_{\text {total }}>101 \mathrm{MeV}$ & +0.04 & 0.02 \\
Uncertainty of the energy calibration & 0.0 & 0.08 \\
$\pi \rightarrow e v$ self-veto in anticounter & +0.03 & 0.02 \\
Time measurement difference for $e$ and $\mu$ & -0.08 & 0.03 \\
Pulse-shape difference for $e$ and $\mu$ & 0.0 & 0.03 \\
Efficiency of $e^{+}$detection in target & +0.15 & 0.01 \\
Subtraction of radiative $\mu$ decay background & -0.45 & 0.17 \\
Subtraction of pion reaction background & -1.13 & 0.06 \\
\hline \hline
\end{tabular}

Gasser and P. Minkowski of the Institute for Theoretical Physics of the University of Berne for many stimulating discussions of theoretical issues. Th. Binkert of the Institute for Applied Physics of the University of Berne gave us useful advice about the timing behavior of plastic scintillators and photomultipliers. The BGO crystals for the calorimeter were developed and manufactured by the company CRISMATEC in Gieres, France. The experiment was supported by the Swiss National Foundation and by PSI.

[1] M. Ruderman and R. Finkelstein, Phys. Rev. 76, 1458 (1949).

[2] S. M. Berman, Phys. Rev. Lett. 1, 468 (1958).

[3] T. Kinoshita, Phys. Rev. Lett. 2, 477 (1959).

[4] W. J. Marciano and A. Sirlin, Phys. Rev. Lett. 36, 1425 (1976).

[5] T. Goldman and W. J. Wilson, Phys. Rev. D 15, 709 (1977).

[6] W. J. Marciano (private communication).

[7] H. L. Friedman and J. Rainwater, Phys. Rev. 84, 684 (1951).

[8] T. Fazzini et al., Phys. Rev. Lett. 1, 247 (1958).

[9] G. Impeduglia et al., Phys. Rev. Lett. 1, 249 (1958).

[10] H. L. Anderson et al., Phys. Rev. 119, 2050 (1960).

[11] E. Di Capua et al., Phys. Rev. 133, B1 333 (1964).

[12] Particle Data Group, Phys. Lett. B 239, 1 (1990), p. VII.2.

[13] D. A. Bryman et al., Phys. Rev. Lett. 50, 7 (1983).

[14] D. I. Britton et al., Phys. Rev. Lett. 68, 3000 (1992).

[15] S. S. Dietrich and B. L. Berman, At. Data Nucl. Data Tables 38, 199 (1988). 